\title{
POSITIVISMO Y SOCIOLOGFA: EN TORNO A UNOS ARTICULOS RECOPILADOS POR ANTHONY GIDDENS
}

\author{
José Jiménez Blanco \\ (Universidad Autónoma de Madrid)
}

En este artículo se realiza una revisión crítica de la colección de textos presentada por Anthony Giddens bajo el título de Positivism and Sociology (Londres: Heinemann, 1974). El autor se refiere más extensamente a los textos de Max Weber, Alfred Schutz y Harold Garfinkel. La revisión ofrece un diálogo intelectual no sólo con estas tres formas de pensar sociológico, sino con la labor compiladora e interpretativa (sobre todo en la introducción) de Giddens. 
Con el título que lieva este artículo, nos presenta el últimamente prolífico sociólogo inglés Anthony Giddens una serie de textos de diferentes autores, todos ellos importantes (los autores) e interesantes (los textos). Lo que pasa es que esta colección de artículos podría haberse titulado de mil maneras diferentes, una de las cuales puede ser sin duda Positivism and Sociology (Londres, Heinemann, 1974), pero no desde luego la única ni la que da más idea a primera vista del contenido del libro. Todo parece indicar un título puesto por el editor, no por el autor.

Los attículos de que consta el libro se pueden agrupar por temas. Un primer tema serían los conceptos weberianos de «objetividad» y «racionalidad»; se inicia con un texto de Max Weber no traducido al castellaro, que yo sepa, donde al hilo de una polémica con Roscher y Knies se establecen nuevas precisiones y matices de pensamientos metodológicos weberianos más conocidos por su versión de Economía y Sociedad. Completa este primer tetna un artículo polémico de Alfred Schutz sobre «objetividad» y «subjetividad» precisamente en Max Weber, y otro artículo, basado fundamentalmente en el pensamiento de Schultz, escrito por Harold Garfinkel, sobre la «racionalidad» en la investigación social. Los tres artículos constituyen un tema no sólo en el sentido de que se sucedan en el tiempo en torno a una misma problemática, sino incluso porque se inicia con el texto de Weber, continúa Schutz sobre textos de Weber, y le sigue Garfinkel, sobre textos de Schutz, que presuponen (e incluso se cita) el hotizonte de problemas metodológicos abiertos por la obra de Max Weber. Este que hlamamos primer tema va a ser el objeto de nuestro comentario crítico en este trabajo.

Nos vamos a detener en este primer tema, porque aunque el pensamiento de Weber, en sus rasgos fundamentales, sea perfectamente conocido por los lectores de lengua castellana, el texto de Weber que se aporta aquí es «nuevo» en el sentido de que no se encuentra traducido (tampoco lo estaba en inglés) y aporta -como hemos dicho- precisiones y matizaciones, que nos ayudarán a profundizar un poco más en el conocimiento de 
Weber, cuya figura como sociólogo se agiganta a medida que pasa el tiempo, y ello aunque no sea susceptible de ser lanzado como titulado «El nuevo idealismo. - Causa y significado en las ciencias sociales», y otro de Herbert Marcuse», «Sobre ciencia y fenomenología». De ellos diremos algo, aunque -a pesar de sus títulos- quedan al margen de los temas agluti. nantes de los textos recopilados por Giddens.

\section{Introducción}

Precede a la colección de textos una Introducción del recopilador, Anthony Giddens, que tiene la virtud de situar el tema en el contexto valorativo (por no decir «apasionado») con que todavía se debate el problema del positivismo y la sociología. «La palabra "positivista", como la palabra "burgués", han llegado a ser más un epíteto peyorativo que un útil concepto descriptivo y, en consecuencia, ha sido ampliamente despojado de cualquier significado aceptado que alguna vez pudo haber tenido» - - co. mienza Giddens (pág. IX, Prefacio). Ya en la Introducción insiste: «EI término se ha convertido en algo de oprobio, y ha sido utilizado tan vaga y ampliamente como un arma de ataque crítico, tanto en filosofía como en sociología, que ha perdido cualquier pretensión de tener un significado aceptado y estandardizado" (pág. 2). Reconozcamos que tiene mérito que en esta situación Giddens comience por ofrecer un concepto de "positivismo». Distingue entre el positivismo en filosofía y en sociología. «El positivismo en filosofía en cierto sentido se resuelve en la aseveración, o la presunción implícita, de que las nociones y afirmaciones de la ciencia constituyen un marco de referencia en que puede set determinada la naturaleza de cualquier forma de conocimiento» (pág. 3). «El positivismo en sociología puede estar ampliamente representado como dependiendo del aserto de que los conceptos y métodos empleados en las ciencias naturales pueden ser aplicados para formar una "ciencia del hombre" o una "ciencia natural de la sociedad"» (ibidem). Para Giddens, el positivismo en sociología ha sido «importado» de la filosofía, y sobre esta base establece los puntos que, según él, constituyen la «actitud positivista» en sociología. Veámoslo.

El primer punto se refiere a que «los procedimientos metodológicos de la ciencia natural pueden ser directamente adaptados a la sociología» (ibidem); subrayado, A. G. Pero añade - lo que nos parece altamente discutible-: «Según este punto de vista, los fenómenos de la subjetividad humana, de la volición o del deseo, no constituyen ninguna barrera parti- 
cular para el tratamiento de la conducta social como un "objeto" parejo con los objetos del mundo natural» (pág. 4).

De lo dicho sobre este libro y su contenido se desprende, efectivamente, que es discutible el título que lleva, al menos parcialmente, pero lo que está fueta de toda discusión es -repito- la importancia de los autores seleccionados y lo interesante (me atrevería a decir también, «actualidad») de los textos seleccionados.

Si con esto se quiere decir que el positivismo pretende obtener un conocimiento objetivo, según el modelo de las ciencias naturales, prescindiendo de lo subjetivo, y buscando su objeto en otras entidades como «la sociedad» o «la Humanidad», estamos de acuerdo. Peto si lo que se quiere decir es que el positivismo de alguna manera hace de lo subjetivo un "objeto" semejante a los de la naturaleza, no estamos de acuerdo. El positivismo, en el mismo Comte, empieza por no incluir en el sistema de las ciencias a la Psicología, por considerar que lo subjetivo del hombre no puede ser objeto de conocimiento científico. Esta noción que tiene su punto de partida en Comte, se sigue en el pensamiento de Durkheim en cuyos "hechos sociales» no cabe lo subjetivo humano; expresamente al buscar el objeto de la sociología intenta acotar "otro objeto de la naturaleza» - los "hechos sociales»- como objeto que le permita superar lo subjetivo como cientificamente intratable. Más recientemente, en la misma línea que podemos llamar francesa, Lévi-Strauss pretende «disolver» al hombre en la naturaleza, como condición indispensable para que se pueda hablar de una ciencia social. Por otra parte, la interptetación althusseriana del pensamiento marxista insistiendo en los aspectos estructuralistas, frente a las interpretaciones «humanistas», nos coloca en la misma dirección. iMita por dónde el pensamiento de Marx resulta coincidir en algo con el pensamiento francés de la tradición positivista! Hablando en serio, no creo que sea sostenible la afirmación de Giddens de que el positivismo considera la subjetividad humana como un «objeto» semejante a los del mundo natural.

Me resisto a creer que haya establecido este primer punto para ofrecernos un concepto de positivismo, en estos términos, que justifiquen la presencia de lo que hemos llamado el primer tema del libro: la polémica de lo «objetivo» versus lo «subjetivo», y la polémica de lo "racional» versus lo «irracional», a partir de Max Weber. Lo que quiero dejar bien sentado desde el principio es que la línea alemana que se inicia con Dilthey, los neokantianos, Windelband y Rickert, alcanzando su forma más acabada en la obra de Max Weber, es claramente antipositivista, precisamente por renunciar el positivismo a dar entrada en el conocimiento de la realidad social a lo subjetivo humano. Lo subjetivo humano entra en las ciencias 
sociales de la mano principalmente de Weber, con toda la tradición alemana que lo precedió, en posición decididamente antipositivista.

El segundo punto de la llamada por Giddens «actitud positivista» se refiere a que «el resultado de las investigaciones puede ser formulado en términos paralelos a los de la ciencia natural: es decir, que la meta del análisis sociológico puede y tiene que ser formular generalizaciones de tipo "leyes" o "cuasileyes" de la misma clase que las establecidas en relación con la realidad natural» (pág. 4). Si el primer punto consideraba al sociólogo como un observador de la realidad social, el segundo punto lo contempla como un analista o uintérprete» de su objeto.

El tercer punto afirma: "Que la sociología tiene un carácter técnico, proporcionando conocimiento que es puramente "instrumental" en la forma; dicho de otra manera, que los resultados de la investigación sociológica no conducen a ningunas implicaciones dadas lógicamente para la política práctica (practical policy) o para la búsqueda de valores. Ia sociología, como ciencia natural, es "neutral" con respecto a los valores." Todo esto es discutible, pero lo es más cuando concluye: «A partir de este aspecto, el positivismo asume un punto de vista específico sobre el sociólogo cono prácticamente implicado en el orden social» (ibidem, subrayado de A. G.).

¿Dónde quedó el vois-pour-prevoir y el prevoir-pour-pouvoir, todo ello inequívocamente comtiano y, por tanto, positivista? Es ignotar demasiado establecer como un punto definitorio de la «actitud positivista» su desvinculación de implicaciones de política práctica; lo contratio está en la letra y, sobre todo, en la última intención de todo el intento intelectual comtiano, para quien quiera entenderlo. $Y$, ¿qué decir del llamado «sociologismo» durkheimiano, también en el ámbito del positivismo?

Lo de que la sociología es «neutral» ante los valores para el positivismo tiene el inconveniente de que: 1) la famosa disputa sobre los valores, en que esta cuestión alcanza su debate más decisivo y en la que es Max Weber quien defiende la neutralidad ante los valores, es posterior a Comte y al positivismo en algo así como 70 años (véase Ralph Dahrendorf, Sociedad y Libertad, Madrid: Tecnos, 1971, págs. 36 y ss.), y, en cualquier caso, se produce fuera del ámbito del positivismo. 2) Como el paladín de la neutralidad valorativa es Max Weber, acaso to que se está insinuando por Giddens es que Weber es positivista; la sospecha toma cuerpo porque es la segunda nota de la «actitud positivista» en que Max Weber parece ser liamado a la parte. No voy a perder un minuto en rebatir esta afirmación. Max Weber no es un positivista. Punto.

Por lo que se refiere a la afirmación, de Giddens, de que el sociólogo, en el positivismo, está prácticamente implicado en el orden social, puede 
querer decir dos cosas: una, en contradicción con lo que acababa de decit antes, que la sociología tiene implicaciones para la política práctica, lo cual creo que es apropiado si se refiere al positivismo. Otra, que el sociólogo forma parte del orden social establecido (si entendemos por ello lo de "prácticamente implicado"), en cuyo caso tendríamos que recordar algunas cosas, que pertenecen a la «pequeña historia» de la sociología, y que no voy a tratar aquí.

\section{Max Weber}

El texto de Max Weber es, desde nuestro punto de vista, una combi. nación de afirmaciones metodológicas ya conocidas, y una serie de matices y precisiones, que surgen al hilo de la polémica con Roscher y Kries, en que se trata de aclarar si lo «subjetivo", como opuesto a lo «objetivo», no lleva consigo la renuncia a la "racionalidad» $y$, en consecuencia, el predominio de lo «irracional» en las realidades humanas y sociales. Como en el texto se combina lo ya conocido con lo nuevo - es decir, Ias nuevas matizaciones y precisiones - nos veremos obligados a tener que presentar unidos ambos aspectos, en gracia del universo de discurso del texto.

Empecemos con una larga, pero inexcusable cita de las palabras de Weber, que son las bases de toda la argumentación: «Siempre que "com. prendemos" una acción humana como condicionada por "fines" claramente conscientes y deseados, y siempre que clatamente teconocemos los "medios" utilizados por el actor, esta comprensión alcanza indudablemente un alto nivel de "evidencia". Si, sin embargo, ahora preguntamos por las bases de esto, en seguida aparece que ello descansa en el hecho de que la relación entre "medios" y "fines" es racional, y como tal es particularmente a propósito para establecer observaciones causales específicas de una naturaleza general que asume un carácter de "cuasiley". No existe ninguna acción racional sin la racionalización causal de aquella parte de la realidad que se encuentra implicada como objeto y como medio de manipulación, es decir, sin estar integrada en un complejo de reglas empíricas (Erfabrung. sregeln) que establece lo que podemos esperar como resultado de un curso dado de la conducta» (pág. 23).

Hechas estas declaraciones, Weber se enfrenta con los que, según él, «erróneamente» afirman que la "comprensión teleológica» es el reverso de un proceso "causal», aunque adrnite - lo que no se relaciona muy claramente con lo anterior- que: 1) «es correcto decir que sin la creencia en la verificabilidad de las reglas empíricas ninguna acción basada en la evaluación de los medios para un resultado intentado puede tener lugar». 
Y 2) «es correcto decit, asociado con lo anterior, que dado un objetivo claramente definido, la elección de los medios no es necesaria e igualmente clara» (ibidem). No obstante todo esto, la cuestión de si la «comprensión teleológico» es el reverso de un proceso causal queda sin demostrar. Se ha manejado la palabra evidente, pero en cuestiones metodológicas conviene demostrar lógicamente lo que se afirma. Suponemos que los razonamientos que siguen pretenden contestar a esta cuestión. Es decir, vamos a realizar con el texto de Weber una "comprensión teleológica", tratando de encajar en el esquema «medios-fines» los argumentos que se esgrimen para la finalidad de demostrar que la "comprensión teleológica» es un proceso «causal».

Veamos el paradigma de la argumentación: «La interpretación racional puede adoptar la forma de un juicio condicional de necesidad» (página 24). Esquema: dado un fin $X$ y de acuerdo con las reglas empíricas conocidas, para alcanzar ese fin el actor tenia que escoger el medio $Y$, entre los respectivos medios $y, y^{\prime}, y^{\prime}$ ". "Esto coincide con una "evaluación" teleológica de la acción que se ha establecido empíricamente» (ibidem). Esquema: la elección del medio $y$, según las reglas empíricas conocidas, garantiza una mayor oportunidad de alcanzar el fin $X$ que la que proporcionan los medios $y^{\prime}$, e $y^{\prime}$ ". "Una elección es "más finalista" que la otra; o se pudiera incluso decir: sólo ella es "eficaz"» (ibidem). Como observará el lector, la argumentación se ha desplazado, de momento, desde el problema de la «causalidad» al problema de la «racionalidad»; puede que lo segundo sea condición de lo primero.

En base al paradigma anterior, Weber sostiene que «Una vez que lo que realmente ocurre es reconocido, esta "evaluación" aparece simplemente como una hipótesis o una construcción de tipo-ideal: la conducta efectiva es comparada con una acción racional. Esto es lo que la acción racional es, de acuerdo con la tegla causal general de la experiencia (reglas empíricas), si uno lo considera "teleológicamente"》 (ibidem; subrayado nuestro). De todo lo anterior se deduce que: 1) las reglas empiricas son causales; 2) las reglas empíticas son racionales; 3) el encajar una acción humana en el marco de referencia de las reglas empíricas proporciona al mismo tiempo la «evaluación» de la acción como racional y su inserción en un proceso causal.

Ahora bien -y es una precisión importante--, las reglas causales generales de la experiencia (reglas empíricas), que garantizan la causalidad y ta racionalidad de la acción humana, se ha dicho: 1) que son verificables, y 2) que tienen el status lógico de una hipótesis o una construcción de tipo-ideal. Es decir, sirven para comparar la acción efectiva con el «tipoideal» o «hipótesis» que puede poner de manifiesto si esa acción efectiva 
encaja o no en el proceso de causalidad y en la evaluación teleológicaracional de las reglas empíricas.

Dos cuestiones se presentan: 1) ¿cómo se construyen esos tipos-ideales o hipótesis? A esto nos contestará Weber más adelante: «Las hipotéticas interpretaciones que nos ofrece nuestra "comprensión" intuitiva son verificadas por nosotros a la luz de la "experiencia"» (pág. 30) y 2) ¿cómo se verifican los tipos-ideales o hipótesis? A la luz de la «experiencia» equivale a encerrar todo el procedimiento en un círculo vicioso. Esquema: tenemos una acción $X$ que queremos «comprender», la comparamos con el tipoideal $Z$, que ha sido construido desde una «comprensión intuitiva» hasta su «verificación por la experiencia», pero es que justamente lo que proporciona la acción $X$ es «experiencia», que puede o no estat de acuerdo con el tipo-ideal $Z$. Si lo está, verifica la regla empírica (que supone causalidad y racionalidad). Si no lo está, ¿qué sucede?, ¿que no verifica el tipo-ideal o que la acción analizada no es ni racional ni causal? Quede planteado aquí el problema. Volveremos sobre ello en un punto próximo.

Weber concluye esta exposición de los rasgos fundamentales de su metodología, haciendo hincapié en que en ringún caso «procedemos a un análisis "psicológico" de la personalidad por medio de algún raro modo de investigar, sino, ayudados por nuestro conocimiento nomológico, a un análisis "objetivo" de una situación dada» (ibidem). Recordaremos esta precisión cuando entremos en la crítica de Schutz.

Entrando en otros detalles que perfilan su pensamiento, Weber apunta que "Aquí, entonces, la "interpretación" se reduce al conocimiento general de que se puede actuar "finalistamente", pero esto significa actuar sobre la base de las consideraciones de las diferentes "posibilidades" de un acontecimiento futuro, de tal manera que se tenga en cuenta diferentes modos concebibles de acción (o abstenciones ante la acción). A causa de la significación eminentemente fáctica de la acción "finalista" en la realidad empírica en este sentido, la racionalización "teleológica" puede ser utilizada como un medio constructivo para la creación de modelos cognitivos ( $\mathrm{Ge}$ dankengebilden). Éstos tienen un valor heurístico poco corriente para el análisis causal de relaciones bistóricas» (págs. 24-25). Estos modelos cognitivos se pueden utilizar, en primer lugar, para analizar acciones de clase puramente individual: son «hipótesis interpretativas relacionando concretas relaciones únicas» (pág. 25). Ejemplo: si queremos conocer la construcción de la política de Federico Guillermo IV, basándonos, de una parte, en ciertos fines que se presumen y, de otra, en la constelación de sus «grandes poderes», eillo sirve -a través de un modelo cognitivopara la finalidad de medir su política real sobre la base de su grado de contenido racional y, en consecuencia, reconociendo los elementos racio- 
nales (en relación con el fin) y los elementos no-tacionales de su acción política efectiva. Es más, aparte de la estimación de los elementos racio nales y no-racionales, puede alcanzarse una clasificación válida de Ia «personalidad" de Federico Guillermo IV «como un factor causal en el con* texto histórico» (ibidem).

De ahí se salta - porque entiendo que hay una «salto» lógico que no se explica- a la declaración de que estos modelos cognitivos pueden set "construcciones de tipo-ideal de un carácter general, como las "leyes" de la economía política abstracta en que, bajo la presunción de una conducta estrictamente racional, se construyen especulativamente ciertos conjuntos imaginados de condiciones económicas» (pág. 25). Se otorga, pues, a estos modelos cognitivos (fundamentalmente, «hipótesis» y «tipos-ideales») la posibilidad de hacer «comprender» tanto casos únicos como fenómenos generales: desde la política de Federico Guillermo IV hasta las «leyes» de la economía política.

Hemos traído hasta aquí al lector, porque creo que el procedimiento metodológico weberiano nos lleva a una situación-límite. En efecto, si el tipo-ideal sirve para interpretar la acción social humana sobre la base de su racionalidad y causalidad mediante un esquema de medios-fines, encajados en unas reglas empíricas validadas por la experiencia, este procedimiento no puede tener la pretensión de ayudar a «comprender» por igual el «caso único» que la «ley» general. En el supuesto del «caso único» (Ia política de Federico Guillermo IV) el tipo-ideal, si logra «comprender» la acción humana, lo será sobre la base de lo que se llama en metodología una «interpretación ad hoc», lo cual queda muy por debajo de las pretensiones científtcas weberianas. $Y$ si el tipo-ideal no logra "comprender» el «caso único» es un disparate puro y simple. ftem más, el proceso que Ileva a la construcción del tipo-ideal para el «caso único», ¿en qué se diferencia del análisis efectivo que se quiere hacer para "comprenderlo»? Sería ridículo acercarse a intuir lo que será después el tipo-ideal, con la posterior verificación de las reglas empíricas mediante la experiencia, cuando ya el hecho de acercarse a intuir puede proporcionar esa experiencia que después está llamada a ser tan útil. No veo manera empírica de diferenciar la construcción del tipo-ideal sobre una acción y su «comprensión» mediante él, de la misma facticidad de la acción. Esto para el «caso único».

Respecto de la «ley» o «cuasiley» general, el tipo-ideal jamás puede llegar a formularlas. Personalmente pensamos que esto no es posible por muchas razones, pero vamos a atenernos al problema tal y como lo ha planteado Max Weber. Esquema: la acción efectiva se compara con el tipo-ideal (modelo cognitivo que trata de comprender la acción como una relación de medios-fines, que si está correctamente construido, nos permi- 
tirá "comprender» la posible tacionalidad de la acción). De una comparación de esta clase sólo puede desprenderse que la acción efectiva está más próxima o más lejana del tipo-ideal. ¿Y qué quiere decir ello? ¿Que la acción es más o menos finalista (respecto de la relación causa-efecto) y por ende más o menos racional? Si hemos entendido correctamente el pensamiento de Weber, cualquier intento de «comprensión» de una acción efectiva puede constituir una prueba de experiencia de las reglas empíricas. Lo que significa que todas las experiencias deben estar de acuerdo con las reglas empíricas para su validez y, en consecuencia, para la validez de la relación de causa-efecto propuesta por el tipo-ideal. Estas relaciones causaefecto validadas por la experiencia son las que pueden proporcionar no ya sólo la formulación de "leyes» o «cuasi-leyes», sino también la «evidencia» que Weber pretende para las acciones «comprendidas» mediante tiposideales. En este sentido, tiene un carácter muy secundario el grado de racionalidad que puede poner de manifiesto la comparación de la acción efectiva con el tipo-ideal. En realidad, lo que pone de manifiesto es si se cumple o no la relación causa-efecto prevista. Y ésta o se cumple o no se cumple. No hay cuestión de grado (como en la tacionalidad).

Como lo que se espera normalmente --según el mismo Weber- es que el tipo-ideal permita comparaciones de la realidad efectiva de la acción con el modelo cognitivo que representa, para alcanzar una mayor o menor «comprensión de las acciones, resulta empíricamente imposible que todas las acciones humanas estén siempte de acuerdo con el tipo-ideal. Por supuesto, Weber no pretende eso de los tipos-ideales; sobre ello volveremos después. Pero, en cualquier caso, la «comprensión» que proporciona una medida del grado de racionalidad de una acción m-en el eje «medios. fines» = "causa-efecto»- nunca será la base para una verificación total por la experiencia de la relación causa-efecto. $Y$ sin ello no son posibles ni «leyes» ni «cuasileyes» ni «evidencias».

Estas limitaciones del procedimiento propuesto por Weber nos las va a presentar a continuación, no como tales limitaciones -por no decir, imposibilidad de alcanzar el objetivo que se proponen-, sino como «no tas» que caracterizan el mismo procedimiento. Son algo así como las uvas verdes de la zorra. Centremos la discusión en un punto: el status metodológico de los tipos-ideales o modelos cognitivos como hipótesis. «Estos esquemas de interpretación - comienza- no son simplemente, como se ha dicho, "hipótesis" análogas a las "leyes" científcas hipotéticas. Pueden funcionat como hipótesis en un sentido heurístico para la interpretación de acontecimientos concretos» (pág. 26). ¿En qué consiste la diferencia? Ésta puede set la contestación weberiana: «Si una "ley de la naturaleza" hipotética fracasa definitivamente en un solo caso, colapsa de una vez y 
por todas como hipótesis. Las construcciones de tipo-ideal de la economía política, sin embargo, no pretenden - correctamente entendidas- una validez general, mientras que una "ley de la naturaleza" tiene que tener esta pretensión si no quiere perder su significación» (ibidem). Me pregunto -dejando aparte el problema de la racionalidad-si una vez que se propone una hipótesis de una relación de causa-efecto es posible afirmar que no pretende validez general. $O$ la relación se da o no se da. $Y$ esto es exactamente igual para las ciencias naturales que para las sociales. Otra cosa puede ser renunciar a establecer relaciones de causa-efecto, pero una vez que se les ha dado entrada hay que someterse al procedimiento de su verificación, y to mismo en las ciencias naturales que en las sociales; si la hipótesis fracasa una sola vez colapsa como tal hipótesis. (Cuestión relacionada, aunque diferente, es la de la probabilidad, pero ahora no podemos entrar en ello.)

La diferencia que estamos examinando entre las hipótesis de las ciencias naturales $y$ las de las ciencias sociales, encuentra una formulación muy hábil, aunque muy discutible, en las siguientes frases: «Una así llamad̉a ley "empírica" en el fondo es una tegla empíricamente validada, que tiene una interpretación causal problemática. Un esquema teleológico de acción racional, de otra parte, es una interpretación con una problemática validación empírica», y se añade: «Las dos son, pues, polos lógicamente opuestos. Pero ambos esquemas son "construcciones conceptuales de tipo-ideal", (ibidem).

A tenor de lo que acabamos de transcribir nos preguntamos: ¿Cómo es posible que una interpretación sea válida si se apoya en una relación causa-efecto que tiene «una problemática validación empírica»? $\mathrm{Si}$ la validación empírica de la relación causa-efecto no se da, ¿en qué se basa la seguridad de que la interpretación es correcta? A riesgo de ser tachado de pecado, permítaseme que reconstruya el discurso weberiano. Para «comprender» una acción bumana hay que encajarla en un esquema medios-fines, que se establece sobre la base de unas reglas empíricas, que siendo intuidas en principio son después validadas por la experiencia. Este procedimiento conduce de suyo a que cada intento de «comprender» una acción es, al mismo tiempo, un intento de interpretación $y$ una prueba de la validez de las reglas empíricas. Si unza sola vez la acción analizada no puede ser «comprendida» por el modelo cognitivo (tipo-ideal) propuesto para el caso -individual o general - todo el tinglado se viene abajo, y de nada sirve que se diga que no pretende "validez general», que tiene un alto nivel «interpretativo», etc. ¿Cómo se puede decir que «comprendemos» una acción si la "comprensión» se basa en unas reglas empíricas a las que no responde la acción? 
A mi modo de ver, el problema consiste en que se ha dado un «salto» lógico no «explanado», si se me consiente la palabreja, entre la relación medios-fines y la relación causa efecto, con el aditamento tampoco «explanado» de que la acción que encaja en esas relaciones es «racional» y, por tanto, puede ser «interpretada» y «comprendida». Pero si la relación medios-fines se encuadra en unas reglas generales de la experiencia (reglas empíricas) que tienen que ser verificadas por la experiencia empírica, cada vez que estamos analizando una acción estamos verificando las reglas empíricas, y si ellas no pueden proporcionar una validez de tales reglas, no se puede decir sobre ninguna base - ni lógica ni empírica- ni que la acción ha sido "comptendida», ni que ha sido «interpretada», ni mucho menos que ha sido «explicada» (es decir, que tesponde a la prevista rela. ción causa-efecto). Personalmente creo que las ciencias sociales ganarían mucho si prefrriesen «explicar», en lugar de «comprender», sobre todo cuando el intento de «comprender» oculta una «explicación» dudosa o inexistente («problemática validación empírica», hemos leído).

El problema se hace particularmente agudo cuando el intento de «com. prensión» se solapa con una base no confesada de intento de "explicación». Es decir, en la medida en que la "comprensión» se alcanza a través de un modelo cognitivo que incluye relaciones causa-efecto, lo primero que hay que hacer es verificar esa relación (y entonces tendremos la «explicación»), y si tratándose de acciones humanas queremos llegar a la «comprensión», bienvenido sea el intento. Lo que no es posible es pretender que se ha logrado una "comprensión» cuando la «explicación» es problemática. Al menos, esto es lo que deduzco del procedimiento weberiano tal como aparece en los textos que estoy comentando. En coherencia con estos comentarios míos, pero en contradicción con lo que ha dicho anteriormente, Weber reconoce que «las disciplinas empíricas que trabajan con la categoría de causalidad y que tratan de aspectos de la realidad -a las que pertenecen la historia y todas las ciencias "culturales", de cualquier clase-m. utilizan esta categoría en su total implicación desde el principio hasta el fin. Elias buscan condiciones y cambios de la realidad como "causas" y "efectos", y tratan en parte de desarrollar "reglas" de "causación" a través de la abstracción que procede de circunstancias concretas, y en parte "ex. plicar" circunstancias concretas "causales" relacionấndolas con aquellas "reglas"» (págs. 29-30).

Siguiendo el discurso weberiano, y supongo que en polémica con los autotes a los que replica el texto, empieza a elucidar el problema de la «racionalidad» versus la «irracionalidad». En este sentido, comienza diciendo que «somos capaces de arrojar luz sobte el postulado de la irracionalidad específicamente empírica de la "personalidad" y de la "libertad" 
de acción» (pág. 26). Entramos en el problema del «libre albedrío» y las ciencias sociales, problema que hoy nadie discute ni parece interesante. Pero lo que dice Max Weber al respecto lo considero importante, y tiene tanto valor ahora como cuando se escribió; en realidad, es el tema central del trabajo, cuyo título original es «Rocher und Knies und das Irrationalitätsproblem», publicado en Wissenschaftslebre.

Lo que dice Weber sobre este problema me parece particularmente atinado, congruente con todo su pensamiento, y estando de acuerdo con él, me voy a limitar a exponer la posición de Weber en esta cuestión, sobre todo porque nos va a ser necesario tener que tecotdarlo cuando entremos en el análisis del texto de Garfinkel.

El problema se presenta así: los autores de referencia parece que han sostenido que el «libre albedrío» humano hace imposible el análisis causal y, por tanto, no tiene sentido la pretensión de una «comprensión» que implique «racionalidad»; «irracionalidad» es más bien lo que parece desprenderse del «libre albedrío». Weber contesta categóricamente: «Lo "más libre", es decir, lo más basado en las "propias" consideraciones del actor - no entorpecido por coerción "extraña" ("externa") o irresistible "afecton - que el actor toma una decisión, lo más enteramente - ceteris paribus- pueden las motivaciones ser analizadas en términos de las categorías de "fines" y "medios", y lo más completamente, en consecuencia, puede ser categotizado y analizado en términos de un esquema de acción racional» (pág. 26). Y al contrario: "A mayor rol jugado por el conocimiento nomológico, sin embargo - para el actor, de una parte, y para el investigador analista, de la otra- más "determinado" es el primero en relación con los "medios" » (pág. 27). Insistiendo en el mismo tipo de considetaciones, afirma: «De hecho, a "más libre" la acción, en el sentido utilizado aquí, es decir, lo menos toma la forma de una "ocurrencia natural", lo más aquella noción de "personalidad" llega a estar validada, la cual encuentra su "esencia" en la constancia de su relación interior con últimos y definidos "valores" y "significados" vitales, Ai ser perseguidos, éstos se convierten en fines, y por tanto transformados en acción teleológico-racional. A más sea éste el caso, menos sitio hay para cualquier clase de concepción naturalística romántica de "petsonalidad", concepción que paradójicamente busca la cualidad sagrada de la personalidad en una "irracionalidad" basada en lo opaco, indiferenciado, "fundamentación" vegetativa de la vida personal: es decir, sobre las entremezclas de una masa de condiciones psico físicas de temperamento y sentimiento, pero que en modo alguno distingue a la "persona" humana del animals (ibidem).

Aunque no disiento fundamentalmente de estos postulados, quisieta hacer algunas observaciones. Primera, aplaudo el sentido de estos párrafos 
que a la vez que un cántico a la libertad humana son un alegato contra quienes sostienen que el «libre albedrio» impide la existencia de ninguna ciencia social y/o del hombre. Segundo, sostener que una acción es libre porque es capaz de adecuar fines a medios es cosa distinta de formular, aunque sea hipotéticamente, relaciones de causa-efecto. Tercero, que una acción sea «racional» por el hecho de responder a una hipotética telación de medios-fines no me parece probado. Es racional, sin duda, la actitud del investigador en querer encajar la acción humana en el esquema mediosfines, pero esto no prueba que sólo sea racional la acción que se acomoda a esa relación. Max Weber nos pone un ejemplo, que como todo ejemplo -según dice Zubiri-traiciona. El ejemplo es el siguiente: si un industrial en la lucha competitiva de la vida económica decide creer en su «libre albedrío» y no seguir las reglas del mercado, eso será a su costa... «Tieñe que escoger entre su extinción económica o seguir máximas muy definitivas de la conducta económicà (pág. 28). Pero, dy si considetamos otro ejem. plo en que un asesino busca el arma más mortal y segura para cometer un crimen? La acción responde a un impecable planteamiento de medios-fines, pero la acción dif́cilmente puede ser calificada de «racional». Ya sé que con esto planteo la vieja disputa, a la que se referirá Garfinkel en su texto, de que Weber no distingue entre una racionalidad del «formal»y una racionalidad «sustantiva». Algo habrá que decir al tespecto cuando comentemos el texto de Garfinkel.

Cuarto, en la actualidad no sería sostenible ese convencimiento que tiene Weber de que trata con «personalidades» que precisamente porque tienen «libre albedrío» van a actuar de acuerdo con alguna hipotética rela. ción medios-fines que se considera «racional». Su distancia de los aspectos «opacos, indiferenciados, vegetativos» de la personalidad humana no va a ser ya posible a partir de la obra de Sigmund Freud, que Max Weber conoció. Cuando todo eso no es una «romántica y naturalística» concepción de la personalidad humana, sino una frontera abierta al conocimiento científico, ni se puede uno distanciar de ello, ni menos ignorarlo. $\mathrm{X}$ al dar entrada a todo eso en el tratamiento científico de la personalidad, los esquemas medios-fines se complican extraordinariamente, de manera que resulta sencillamente naïve no tenerios en cuenta. Weber no los pudo tener, pero nosotros sí los tenemos que tener, y desde esta perspectiva valorar la propuesta weberiana. Quinto, no ya el esquema medios-fines, sino el que le sirve de base -el de causa-efecto-, ¿por qué tiene que ser «racional» o «irracional»? Weber ha insistido mucho en el valor interpretativo de los tipos-ideales. ¿Por qué no verificar primero las relaciones de causa-efecto empíricamente, desvincular esas telaciones de la de medios-fines y, sobre todo, no atribuir automáticamente el carácter de racionalidad o de irracio. 
nalidad sobre estas bases, dejando para una interpretación menos constreñida a los modelos cognitivos de tipo-ideal y más abierta a lo que permiten, de una parte, la metodología científica, y de otra, las técnicas de investigación actuales?

Sexto, a pesar de todo lo dicho creo que no es posible una ciencia social sin las categorías de «causalidad», «racionalidad» y «libre albedrío». Como se ha visto, no estoy de acuerdo con la solución que Weber propone al manejar estas categorías, pero sí estoy de acuerdo con el fin de fundamentar una ciencia social sobre estas categorias; con lo que no estoy de acuerdo es con los medios propuestos por Weber para alcanzar ese fin.

Hemos resumido, comentado y criticado hasta aquí el texto de Weber, de manera que nos facilite la comprensión de la polémica que va a sostener Alfred Schutz sobre el problema de la «objetividad» versus la «subjetividad», y la que sostiene Karold Garfinkel sobre el sentido de la «racionalidad» en la vida social. Weber concluye su artículo con unas palabras que son un resumen muy fiel de todo lo que ha venido argumentando en el texto. Lo transcribo porque en pocas palabras encierra todo lo que es el pensamiento de Max Weber sobre los problemas que hemos ido desarrollando al hilo del discurso del mismo Weber. Dice así: «La creencia de que, en cualquier zona del conocimiento, los postulados deterministas incluyen el postulado metodológico de establecer categorías y "leyes" como la única pretensión, constituye tan gran error como la presunción que sostiene lo contrario: que una creencia metafísica en el "libre albedrío" debe excluir la aplicación de categorías y "reglas" para la conducta humana. No existe ninguna base para la creencia de que el "libre albedrío" humano tiene que estar conectado con una específica "incalculabilidad" o en cualquier manera con cualquier clase específica de irracionalidad "objetiva" de la acción humana. Hemos visto que lo contrario es lo que acon. tece» (pág. 31).

\section{Alfred Scbutz}

Alfred Schutz comienza con la exposición de algunas distinciones weberianas, que se contienen en las primeras páginas de Economía y Sociedad. En concreto, se centra en la distinción entre: 1) de una parte, el tipo de «comprensión» que consiste en «la comprensión observacional direc" ta del significado subjetivo de un acto dado como tal, incluyendo declaraciones verbales». Y 2) el tipo de «comprensión» que «es comprensión racional de la motivación, que consiste en situar el acto en un contexto de significado inteligible y más incluyente» (pág. 33). Un examen muy 
atento de estos dos tipos de «comprensión» constituye el contenido del artículo de Schutz, cuyas continuas citas y referencias a Husserl hacen difícil saber cuando está hablando Schutz y cuando Husserl.

Veamos, en primer lugar, lo que tiene que decir Schutz sobre la «comprensión observacional», tanto de «afectos» como de «pensamientos». $\mathrm{Y}$ se pregunta: «¿Cómo podemos llegat a una comprensión del significado subjetivo de estas experiencias a través de la observación directa?» (ibidem). Schutz pone un ejemplo: sería un acto de comprension observacional si yo miro a la cara de $A$ y sus gestos y saco la conclusión de que se trata de una explosión de ira. Pero, ¿cómo saber si lo que estamos mirando es una mera reacción, o se encuentra en línea con lo que puede ser considerado orientado significativamente, o si eilo consiste en una reacción incontrolada de un estímulo excepcional, o si $A$ simplemente ha cogido un bertinche como reacción ante sentimientos reprimidos? Para Schutz, «la observación directa no me da ninguna respuesta a esta cuestión» (pág. 35). Esto por lo que se refiere a la "comprensión observacional» de «afectos».

Para la «comprensión observacionai» de "pensamientos», Schutz nos tiene preparadas unas distinciones de Husserl sobre los «dos diferentes sentidos del significado de un juicio». En primer lugar, tenemos el «contenido del juicio»y, en segundo lugar, la «actitud epistémica». El «contenido del juicio» no cambia, pero la «actitud epistémica»-que es la que la persona que está utilizando o expresando el juicio tiene hacia el contenido del juicio- puede ser muy variada. Y es precisamente esta «actitud epistémica» la que, según Weber (según Schutz), determina lo que la expresión del juicio «significa». Precisamente, esta «actitud epistémica» es «lo que no puede ser determinado por observación directa» (pág. 35; subrayado de Schutz). En resumen, «la comprensión observacional de la conducta externa de otra persona no es claramente suficiente para establecer estos puntos. Éstas son cuestiones de significado subjetivo y no pueden ser contestadas mitando (watcbing) simplemente la conducta de alguien, como Weber parece pensar» (pág. 36). Desde luego estoy de acuerdo, y Weber también lo estaría, en que watching la conducta de nadie se pueda sacar cualquier resultado sobre el sentido subjetivo de Ia acción. Pero antes de Weber, en vida de Weber y después de muerto Weber (el artículo de Schutz se escribió en 1932), en metodología científica «observación» no equivale a «mirar» (la palabra utilizada por Schutz - to watch--- es la que se emplea, por ejemplo, para decir que estoy mirando la televisión). Por «observación», incluso «observación directa», se entiende la utilización de los sentidos ayudados por medios técnicos para corregir y ampliar las posibilidades de los sentidos. Estos medios técnicos pueden ser desde un mictoscopio hasta un cuestionario o un «test», e incluso se puede estimar como medio técnico 
la construcción de un tipo-ideal, contribución otiginal del mismo Weber, que Schutz debió recordar en este contexto. La crítica que se basa en que Weber propone «observar» como simple «mirar», es tan ingenua que revela el desconocimiento de la metodología científica, en generai, y la metodología de Max Weber, en particular. En conclusión, tanto la «comprensión observacional de «afectos» como de «pensamientos» es accesible a Ia observación científica, siempre que no se la confunda con un simple mirar $y$ ver.

Vamos ahora con la «comprensión motivacional». Esta cuestión se concentra, en primer lugar, en demostrar «la imposibilidad de la comprensión motivacional sobre la base de la observación sola» (pág. 36). No hay que molestarse demasiado en seguir la argumentación porque, como hemos visto, está basada en un concepto de «observación» que probablemente tenga el mérito de ser la de Husserl, Schutz y toda la Fenomenología, pero que no coincide con lo que la metodología científica, incluso la metodología de Max Weber, entiende por «observación». AI abordar esta cuestión, Schutz empieza calificando a Weber de «confuso, si no claramente contradictorion (ibidem). Aunque nuestro gusto sería dar por liquidada la crítica a Schutz en este punto, nos vamos a entretener un rato demostrando que el «confuso y claramente contradictorio» es Alfred Schutz. Por otra parte, no hemos entrado todavía en el tema del artículo que es «el significado subjetivo y objetivo".

Entrando en este problema, Schutz va a distinguir, de un lado, el "sentido subjetivo", desde nuestro punto de vista, y de otro, ese sentido que los actos tienen para los que intervienen en ellos. Además, considera esencial el "pasado» y el «futuro» del actor para poder llegar a una «comprensión motivacional». Por último, distingue entre el significado subjetivo para el observador, y dicho sentido para el actor, concluyendo que «hemos mostrado que el motivo de una acción no puede ser comprendido a menos que conozcamos primero el significado de esa acción. Pero el actor es quien tiene este conocimiento, no el observador» (pág. 38). Parece que una de las características de la Fenomenología, más que en distinguir, consiste en desmenuzar cualquier realidad de manera que sea inaccesible al conocimiento científico. ¡Qué duda cabe que existen todos esos puntos de vista -esencialmente, el del observador, el de los actores y, eventualmente, el del actor--, y que el conocimiento será más amplio si abarca el pasado, el presente y el futuro! Lo que ocurre es que con todas esas notas y distingos, el análisis pasa de «un análisis objetivo de una situación dada» -recordemos las palabras de Weber- a un análisis subjetivo de la personalidad del actor, y si se me apura de la personalidad de los otros actores y hasta de Ia personalidad del observador. Esto es justo lo que no quería 
hacer Weber: «un análisis psicológico de la personalidad». No hay inconveniente en que los psicólogos hagan análisis de la personalidad; es más, son ellos los que tienen que hacerlos. Pero la sociología se mueve en otro nivel, precisamente en el del «análisis objetivo de una situación dada».

Por otra parte, se hace necesario recordar aquí algo muy esencial de la metodología científica, y es que --según Zubiri- la ciencia no opera con la realidad empírica en toda su tiqueza, sino con «modelos» que seleccionan ciertas notas de la realidad, que «sustituyen» a la realidad empírica en el trabajo científico. La línea que Schutz está siguiendo, y que se acentuatá más adelante, consiste en una descripción exhaustiva de la realidad empírica considerada, y cuando ha llegado a ese punto -como no podía menos- acaba pontificalmente con el anuncio de que la realidad así entendida no es susceptible de observación. Sino que, puestos en esa línea, la descripción, a pesar de todo, se queda siempre corta, porque siempre habrá algún rasgo más, alguna nota más, que se le había quedado en el tintero al fenomenólogo de turno.

Sin embargo, de esta verdadera pasión de los fenomenólogos por las distinciones, «la distinción de Weber —dice Schutz- entre comprensión observacional y motivacional es arbitraria y sin ninguna base lógica en su propia teoría. Ambos tipos de comprensión se basan en un contexto de significado objetivo. La comprensión del significado subjetivo no tiene lugat en ninguna de ellas» (pág. 39). ¿Le tecordamos a Schutz todo to que hemos examinado en el anterior texto de Weber? Hagámoslo resumidamente. Primero, toda la metodología weberiana se basa en la pretensión de captar el significado subjetivo - que es una de las notas de su concepto de «acción social»- en el esquema medios-fines, que presupone una racionalidad compartida por el actor, los actores y el observador. No se renuncia al significado subjetivo, sino que se trata de «comprenderlo» en un contexto «objetivo» de una situación. Segundo, si no se entiende el sentido («objetivo», me parece) del término observación, la distinción weberiana puede parecer «arbitraria». En otro caso, no. Tercero, la saciologia comprensiva propuesta por Weber precisamente lo que intenta es situar en un contexto objetivo la significación subjetiva que la acción tiene para los actores. $O$ lo que es igual: no se trata de hacer análisis de la personalidad, sino análisis objetivos de situaciones dadas, como ya hemos tenido que recordar varias veces. Es decir, que lejos de no tener base lógica en su propia teoría, esta distinción lo que hace es convertir en «operativos» los enunciados de su teoría. Cuarto, el rechazo de la comprensión de lo subjetivo en un contexto objetivo, sea cual sea la «comprensión» (observacional o motivacional), que parece ser toda la base de la argumentación de 
Schutz, lo vamos a ver pronto contradicho por el propio autor. Lo recordaré cuando llegue su momento.

No obstante lo dicho hasta ahora por Schutz, va a reconocer, por to pronto, una excepción: el dominio de la vida cotidiana. «... existe - diceun punto epistemológico válido subrayando la distinción entre comprensión observacional y motivacional. En la vida cotidiana experimentamos directamente los actos de otro. Interpretamos estos acontecimientos externos que llamamos "acto de otro" como indicaciones de una corriente de conciencia que se sostiene fuera de la nuestra propia. En la medida en que hacemos estas cosas, podemos "comprender" los acontecimientos en cuestión, leyendo las indicaciones tal como ellas acontecen, y así, directamente, presenciar la acción como ella se desarrolla, observándola "en el modo de actualidad". La comprensión observacional es, pues, enfocada hacia la acción según ella tiene lugar, y nosotros, como seres vivientes al lado del actor y compartiendo su presente, particjpamos experimentalmente en el verdadero curso de la acción. En esencia, la comprensión directa u observacional es simplemente la comprensión que ejercemos en la vida cotidiana en nuestra relación directa con otra gentè (págs. 39-40). Maravillosa vida cotidiana que puede convertir en realidad, en lógico y en de acuerdo con las teorías de alguien, lo que hasta ahora ha sido rechazado como arbitrario, confuso, contradictorio y sin fundamento en la teoría de Weber. De momento, ya tiene fundamento en la filosofía fenomenológica. Perdón: en la teoría sociológica de Schutz. Hemos llegado a la conclusión de que la comprensión observacional directa es posible en la vida cotidiana. ¿Y la comprensión motivacional?

Tras algunos tazonamientos de que hago gracia al lector, Schutz concluye que «la "comprensión interpretativa" que define la sociología interpretativa no puede ser comprensión observacional. Más bien, el método científico de establecer el significado subjetivo es la comprensión motivacional, mientras que la clase de comprensión propia de la vida cotidiana es de carácter observacional» (pág. 40). Es decir, que después de una crítica negativa, en que no han escaseado los adjetivos peyorativos, Schutz está de acuerdo en que la comprensión directa u observacional es posible en la vida cotidiana, y que el procedimiento científico de captar el significado subjetivo lo constituye la comprensión motivacional. Creo que Weber estaría de acuerdo. Pero el que no está de acuerdo soy yo.

Primero, no estoy conforme porque Schutz compara un «modo de vida» - la vida cotidiana- con un procedimiento de observación científica. Lo correcto sería distinguir -ahora sí- el «modo de vida» de la vida cotidiana con el de la vida no-cotidiana. Como veremos en el artículo de Garfinkel, sobre textos del propio Schutz, eso es característico de este 
último. Segundo, porque lo que Schutz cree ver en la vida cotidiana de "comprensión observacional» no tiene nada que ver con ningún procedimiento científico. En la vida cotidiana la gente tiene que habérselas con la realidad que le circunda y, por supuesto, ello implica algún tipo de conocimiento sobre los demás - de otra manera setía imposible sencillamente "convivir». Pero este conocimiento -al nivel de la «experiencia» vivida e, incluso, del «sentido común»-, no tiene nada que ver con el conocimiento científico, ni siquiera con el tipo de conocimiento que está implicado en la «comprensión observacional». Para Weber, cualquier «comprensión» supone el instrumento del tipo-ideal, que se interpone entre nuestra experiencia de la vida cotidiana o no-cotidiana y la posibilidad del conocimiento científico. Tercero, si el «modo de vida» de la vida cotidiana bastara para alcanzat — sin interponer recurso técnico observacional alguno- el conocimiento, la ciencia, al menos a ese nivel, sería innecesaria. Bastaría vivir para conocer. Porque esto no es así — ni siquiera en la vida cotidiana-, ha surgido la ciencia social. Cuarto, ¿en qué «momento»o «lugat», por decirlo de alguna manera, sitúa Schutz el punto en que la «comprensión observacional» ya no es posible? Schutz no responde nada. Quede constancia de que el procedimiento de Schutz de comparar la vida cotidiana, de una parte, con la "comprensión motivacional», de otta, es incorrecto. Lo correcto sería, primero, distinguir entre vida cotidiana y vida no-cotidiana y, segundo, aplicar indistintamente los dos tipos de «comprensión» weberianos (el observacional y el motivacional), según el grado de conocimiento que nos sea necesario o accesible de acuetdo con la realidad que queremos investigar. Quinto, reservo para más adelante el problema del tránsito - desde el nivel del «sentido común» hasta el conocimiento científico- de la vida cotidiana a la vida no-cotidiana, como base para una metodología que, partiendo del nivel de la «experiencia», construya modelos cognitivos de tipo-ideal que se verifquen en el terreno de los hechos.

Si el lector ha podido seguir con comodidad estos vericuetos, más bien simplones, aunque con una pedantería pretenciosa, le parecerán atinadas estas palabras que el maestro Arboleya dedicó a la Fenomenología: «Indudablemente, los análisis fenomenológicos han ofrecido un material utilizable, pero no para el fin que ellos se proponían. Pues, en efecto, en primer lugar..., en ei fondo de todo ello está la creencia de que la sociedad se agota en una serie de relaciones putamente interindividuales entre un yo intemporal, un tú intemporal y un él intemporal, que forman un nosotros vivido por el yo, el tú y el él. Nuevamente aquí se extrae al individuo del conjunto social y se subordina éste a la corriente de vivencias de los relatos. Esto de un lado. De otro, se supone que lo vivido es lo comprendido y que lo vivido comprendido es la tealidad) (Estudios de teoria de la socie- 
dad y el Estado, Madrid: Instituto de Estudios Políticos, 1962; pág. 596).

No bien Schutz nos ha embarcado, una vez más, en un mar de distingos y desmenuzamientos de la realidad, nos sorprende dejándonos en la estacada, al decirnos que «es obvio que existen tantos enigmas rodeando al problema del significado subjetivo que en este primer estadio de la discusión apenas se puede esperar alcanzar un claro entendimiento de su naturaleza» (págs. 41-42). Más adelante $-\mathrm{y}$ ya no lo seguitemos-m, Schutz anuncia "más profundos» niveles de «entendimiento de su naturaleza», pero no hay tal. Lo que hay son más y más descomposiciones, desmenuzamientos y distinciones, que si bien se acercan cada vez más a una descripción casi exhaustiva de las realidades que se trae entre manos, por la misma razón cada vez más se apartan de esa «sustitución» de la realidad empírica -de que nos habla Zubiri- por otra más simplificada, accesible a la metodología y técnicas de investigación de la ciencia empírica.

A continuación, Schutz se va a enfrentar con el problema de distinguir wes lo suyo- entre significado subjetivo y significado objetivo. Hasta ahora se ha limitado a considerar como «objetivo» el que no es «subjetivo». Por tanto, un criterio negativo. Veamos el criterio positivo que permita esta distinción. Esquema: sea $M^{1}$ el significado de una acción dada de $A$ para un actor dado $X$. $A$ puede ser observado por $F$ (un amigo) y por $S$ (un sociólogo). Ello supone que la acción de $A$ tiene sentido para ambos observadores. Ambos van a conectar el curso externo de la acción de $A$, que ellos toman como una indicación de las experiencias subjetivas de $X$, con un significado. «Sin embargo - - dice Schutz - ya hemos demostrado que el significado intentado $M^{t}$ que $X$ da a su acción no puede ser descubierto ni por comprensión observacional ni motivacional» (pág. 41). (Se le olvida lo que nos ha dicho antes de la vida cotidiana, pero sigamos adelante.) Lo que sucede es que $F$, sobre la base de su experiencia práctica, interpreta la acción externa de $A$, como teniendo el significado $M^{2}$. Y $S$, sobre la base de una construcción de tipo-ideal, asigna a la acción un tercer significado $M^{3}$. En la terminología de Weber $M^{I}$ sería el significado subjetivo o intentado que $A$ atribuye a su propia acción, $M^{2}$ y $M^{3}$ serían el significado objetivo de este acto. «Pero, después de todo, $M^{2}$ es sólo el significado objetivo relativo a $F$, y $M^{3}$ es sólo el significado objetivo relativo a $S$. En consecuencia, Ilamar a $M^{2}$ y $M^{3}$ contenidos-de-significado objetivos es simplemente decir que son diferentes de $M^{l}$. Como cuestión de hecho, ya que $M^{3}$ sólo se puede inferir de la conducta externa de $A$, el significado intentado tiene que ser considerado como un concepto límite con el que $M^{2}$ y $M^{3}$ nunca coincidirían ni siquiera bajo óptimas condiciones de interpretacions (ibidem).

Continuemos de la mano de Schutz: éste nos va a aclarar el significado 
objetivo de $M^{2}$ y $M^{3}$, mediante una interpretación según la cual $M^{2}$ es el significado subjetivo que $F$ da al acto $A$ de $X$, y $M^{3}$ es el significado subjetivo que $S$ le da. «Semejante lectura equivocaría enteramente lo que Weber tiene en la cabeza cuando utiliza el término "significado subjetivo o inten. tado". Porque es obvio que una acción tiene sólo un significado subjetivo: el del mismo actor. Es $X$ quien da significado subjetivo a su acción, y los únicos sentidos subjetivos dados por $F$ y $S$ en esta situación son los sig. nificados subjetivos que ellos han dado a sus propias acciones, especial. mente, sus acciones de observar a $X$ » (ibidem).

Veamos, por lo menos, ent qué acaba el esquema de $A, X, F, F, M^{I}, M^{2}$ y $M^{3}$. «... el fenómeno del mundo externo tiene un significado no sólo para usted y para mí, para $F$ y para $S$, sino para cualquiera que vive en él. Sólo existe un mundo externo, el mundo público, y se nos da por igual a todos nosotros. En consecuencia, cada acto mío a través del cual yo otorgo al mundo un significado se vuelve a referir a algún acto de otor. gamiento de significado (Sinngebung) de ustedes con respecto al mismo mundo. El significado así constituido es un fenómeno intersubietivo» (pág. 42; subrayado nuestro).

Sencillamente -y para no perder la calma-, recordemos que en la página anterior ha dicho que -lo repetimos- «como cuestión de hecho, ya que $M^{L}$ sólo se puede inferir de la conducta externa de $A$, el significado intentado tiene que ser considerado como un concepto límite con el que $M^{2}$ y $M^{3}$ nunca coincidirian ni siquiera bajo óptimas condiciones de interpretación» (subrayado nuestro). Si el significado es un fenómeno inter. subjetivo, ¿cómo se puede decir que ni por casualidad coincidirían los significados del actor, del amigo y del sociólogo? ¿Quién es el contradictorio, Weber o Schutz? ¿Quién es el arbitratio que una vez nos habla de la «vida cotidiana» y otta vez del «mundo público» y no se molesta en delimitar el sentido de una y otro? ¿Quién es el que en lo que dice no se apoya en su propia teoría, cuando Schutz nos deja en la estacada cuando le viene en gana y como supremo argumento pata no seguir adelante se refiere a que Husserl, en su Lógica formal y transcendental, estableció el problema de la fenomenología del conocimiento, pero en modo alguno lo resolvió?

La cosa acaba así: «Ahora es seguro que las objetividades ideales (ideale Gegenständlicbkeiten) que forma el contenido de significado de las expresiones y de los grandes sistemas del lenguaje, el arte, la ciencia, el mito, etc., de la que son inseparables partes, juegan su papel en la interpretación de cualquiera de la conducta de otras personas. Todas estas interpretaciones presuponen el uso de tales esquemas interpretativos. Esto sirve también para dar cuenta del significado subjetivo observable por $F$ y por $S$ cuando 
la acción $D$ tiene lugar. La interpretación de tales cursos de la acción tiene lugar regularmente de acuerdo con los esquemas que se tienen a mano para empezar, incluso aunque sean seleccionados por $F$ y por $S$ y sean, en consecuencia, relativos a ellos» (pág. 44; subrayado de A. S.). ¿Es necesario recordar la insistencia en el valor interpretativo asignado por Weber a sus «esquemas interpretativos», con el nombre de construcciones de tipo-ideal o modelos cognitivos? Para no tepetir cosas, dejo al lector que repase los comentarios a Weber.

\section{Harold Garfinkel}

El artículo de Harold Garfinkel parece ser algo así como un paper preliminar teórico para un proyecto de investigación subsiguiente. Como un instrumento de trabajo que es, se le pueden perdonat las continuas teferencias a Schutz, aunque hay que preguntarse cuál es la contribución de Garfinkel, aparte presentar las ideas de Schutz, en esta ocasión. Téngase en cuenta que los textos que se citan de Schutz estaban ya previamente traducidos al inglés, con lo que el artículo de Garfinkel no tiene ni siquiera el mérito de «introducir» en inglés el pensamiento de aquél. Ello se inserta en la corriente norteameticana en que no hay tontería que se le haya ocurrido a un europeo que no haya encontrado un profesor americano dispuesto a convertirla en un proyecto de investigación empírica. Lástima que para los americanos Marx haya sido hasta hace poco, de hecho, un autor probibido, porque de no ser así dispondríamos de una contrastación empírica del pensamiento marxista, que buena falta nos hace. Los marxistas americanos hasta ahora han sido meros repetidores de teoría, un Itrjo que la sociedad norteamericana se ha permitido para que no digan.

Garfinkel va a discutir y tomar posición ante el problema de la «tacionalidad». Sólo se cita a Weber al final del artículo, pero es claro que está presente en toda la discusión. Lo está en las primeras líneas, cuando se presenta lo que comínmente se entiende por «tacionalidad» en las ciencias sociales. Así dice: «Los investigadores sociológicos deciden una definición de racionalidad mediante la selección de uno o más rasgos de entre las propiedades de la actividad científica según elia es descrita y comprendida. La definición es entonces utilizada metodológicamente para ayudar al investigador a decidir lo realista, patológico, prejuzgado, desilusionante, mítico, mágico, ritual y rasgos similares de las creencias, pensamientos y conductas de cada día) (pág. 53). Por si la alusión a Weber no estaba suficientemente clara, en nota a pie de página se añade: «Una definición que disfruta actualmente de favor es la conocida como regla 
de los medios empiricamente adecuados. Las acciones de una persona se conciben por el investigador como pasos para el cumplimiento de tareas, cuyo posible y efectivo cumplimiento es empíricamente decible. La adecuación empírica es, pues, definida en términos de las reglas del procedimiento científico y las propiedades del conocimiento que tales procedimientos producen» (ibidem). Obsérvese ya el leve sesgo, que luego se va a argumentar, de atribuir la racionalidad al ptocedimiento metodológico, y no a la tealidad misma.

Coherente con este sesgo, Garfinkel encuentra que es abrumadora la frecuencia con que los sociólogos encuentran que no son interesantes empíricamente las propiedades de racionalidad que los investigadores atribuyen a las acciones humanas. Por lo que concluye: «El resuitado es que en la mayoría de las teorias de la acción social y la estructura social las acciones racionales tienen asignado un status residual» (págs. 53-54). Esto no es verdad. Disponibles están las teorias de Weber o Parsons sobre la accion social y la estructura social. Disponible está la obra de Marion J. Levy, The structure of Society, que cita en sus referencias el propio Garfinkel. En ninguna de ellas la racionalidad tiene un carácter residual. Esto no se putede decir ni de la obra de Pareto, que tanto aportó al análisis de las conductas humanas no-racionales.

Pero como Garfinkel ha partido del carácter residual de la racionalidad en la teoría sociológica, helo aquí dispuesto a emprender una cruzada para «este status residual mediante la teintroducción como un problema para la investigación empírica a) de las varias propiedades racionales de la conducta, tanto como $b$ ) las condiciones de un sistema social en que tienen lugar varias conductas racionales" (pág. 54). Mucho nos hace esperar este planteamiento. Veremos en qué queda.

Como primer paso en esta dirección nos transmite un inventario de los sentidos del término "tacionalidad», debido a Schutz, en que se recogen nada menos que $; 14$ ! sentidos de esta palabra. Sólo voy a transcribir los títulos de los 14 apartados: 1. Categorizar y comparar. 2. Error tolerable 3. Búsqueda de los «medios». 4. Análisis de alternativas y consecuencias. 5. Estrategia. 6. Concern for timing. 7. Predictíbilidad. 8. Reglas de procedimiento. 9. Elección. 10. Bases de elección. 11. Compatibilidad de finesmedios con principios de lógica formal. 12. Claridad y distinción semánticas. 13. Claridad y distinción «por sí mismas». Y 14. Compatibilidad de la definición de una situación con el conocimiento científico (págs. 54-59).

La mera lectura de los epígrafes nos da idea de que nos encontramos con otro de los desmenuzamientos propios de Schutz que ni son una distinción (la mayoría de los epígrafes aluden a contenidos que se solapan) ni llegan a ser tampoco una descripción completa, aunque tal vez sea eso lo 
que se pretende. Debe quedar claro que no se tebasa el nivel de análisis weberiano de la racionalidad, sino en cuestiones cuyo carácter de racionalidad sería harto discutible (como tos epígrafes 6, 12 y 13). Los demás epígrafes se mantienen en los límites de la conceptualización de Max Weber. Por otra parte, suponemos que Garfinkel, en su reintroducción de la categoría de racionalidad, se va a atener a los términos transcritos del inventario de Schutz. Como veremos, no es así. ¿Entonces para qué esta transcripción?

Por lo pronto, para decirnos --sin que medie «explanación» alguna-.que: «Al extender los rasgos de este tipo de conducta para incorporar todas las precedentes racionalidades, surge una distinción entre los intereses de la vida cotidiana y los intereses del teorizar científico» (pág. 60). Otra vez nos encontramos, no con la distinción entre vida cotidiana y vida no-cotidiana (que acaso sea lo que Schutz llamó anteriormente «mundo público»), sino con la contraposición entre vida cotiojiana y procedimiento científico. Efectivamente, el discípulo no es más que el maestro.

Estamos en el punto clave del paper. Su «almendra»-Su «crux», en palabras de Garfinkel- consiste en que «las racionalidades científicas, de becho, tienen lugar como propiedades estables de las acciones y como ideales sancionables sólo en el caso de acciones dirigidas por las actitudes del teorizar cientifico. Por contraste, las acciones dirigidas por la actitud de la vida cotidiana están marcadas por la ausencia específica de estas racionalidades, ya sea como propiedades estables o ya sea como ideales sancionables» (pág. 61; subrayado de H. G.). Nota: Ias «racionalidades científicas» son de los epígrafes 11 a 14.

De ello se sigue que procede distinguir rigurosamente, como dos cosas perfectamente diferentes, lo que llamaríamos «actitud de la vida cotidiana» y «actitud del teorizar científico». La distinción, ¡cómo no!, se establece sobre una descripción de Schutz. Para facilitar al lector el enfrentamiento de las dos actitudes, permítaseme presentarias a doble columna y con la numeración que tienen en el original.

Actitud de la vida cotidiana

$10^{\circ}$
«Establecida como una propiedad de los acontecimientos, esta presunción consiste en la expectativa de que existe una relación de indudable correspondencia entre las apariencias

\section{Actitud del teorizar científico}

«Por contraste, las actividades del teorizar cientiffico se encuentran dirigidas por el extraño (strange) ideal de duda que es en principio ilimitado y que específicamente no 
Actitud de la vida cotidiana

efectivas del objeto y el objeto-intentado-que -aparece-en-esta-determinada-manera. Fuera del conjunto de alternativas entre las apariencias efectivas del objeto y el objeto intentado, como por ejemplo la rela* ción de dudosa cortespondencia entre los dos, la persona espera que la presupuesta correspondencia indudable es la razonable, y que la otra persona utiliza la misma expectativa de una manera más o menos idén. tica.»
En la vida cotidiana, to que la persona conoce uen la manera en que ella lo conoce, ella presume que es parte integral de sí misma como objeto social. Ella sanciona su competencia como un miembro de buena-fe del grupo como una condición para apreciar adecuadamente el sentido de sus asuntos cotidianos; ella no trata esta competencia como irrelevante».
Actitud del teorizar científico

reconoce las estructuras sociales normativas como condiciones que constriñen" (págs. 62-63; el subrayado es de Garfinkel, así como el derroche de guiones, ¿o será tal vez de Schutz?).
«Schutz describe la perspectiva temporal de la vida cotidiana. La persona que se dice que orienta los acontecimientos en la actitud de la vida cotidiana concede a los posibles acontecimientos un acento temporal que se forma a partir de lo que la persona presume que es un esquema de relaciones que él y otras perso-
«Por contraste, las reglas interpretativas de la actitud del teorizar científico sostienen que la exactitud de un modelo tiene que ser probada, suspendiendo en el entretanto el juicio sobre la relevancia de lo que el teórico conoce en virtud de sus disposiciones corporales y sociales en el mundo real» (pág. 63; subrayado de $H . G$.). 
Actitud de la vida cotidiana

nas emplean de una manera equivalente y estandardizada.»
Actitud del teorizar científico

que como un medio de someter a un calendatio sus acciones» (páginas 63.64$)$.
"La persona asume una particular "forma de socialidad". Esta presunción consiste en la creencia de que alguna característica disparidad existe entre la imagen de sí misma que atribuye a los otros y la imagen que ella tiene de sí misma, es decir, una disparidad característica entre su vida pública y privada. Presupone, también, que las aiteraciones de sus características disparidades permanecen dentro de su control anónimo...»
«Esta presunción... se modifica profundamente en las reglas que dirigen las acciones del teorizar científico. En la sociedad del teorizar científico, el teórico se encuentra fuera de un rol. Todos los asuntos relevantes para su presentación del mundo son públicos y publicables. Resulta difícil ver cómo alguien pudiera avergonzar a un teórico científico o indignarlo» (pág. 64).
«La actitud de la vida cotidiana contiene la presunción de un mundo común intersubjetivo de comunicación. Los acontecimientos de la vida cotidiana se encuentran informados por este transfondo integral de sentido.»
«Esta presunción de un mundo de comunicación intersubjetivo se encuentra sorprendentemente modi. ficado en las acciones del teorizat cientifico. Las "otras personas relevantes" para el teórico científico (los colegas, por ejemplo) son en el me. jor de los casos instancias aceptables de altamente abstractos "investigaJotes" que son, en lo ideal, manuales abiertos de maneras propias de proceder» (pág. 65).

Distinguir lo que es diferente en principio no parece ser tarea excesivamente difícil. En efecto: todos podemos estar de acuerdo en que la "actitud de la vida cotidiana» es distinta de la «actitud del teorizar científico». Que yo sepa, nadie ha pretendido nunca que sean una misma cosa. 
Sin embargo, he aquí to fácil convertido en un problema dificultoso. La discusión de los cinco puntos que acabamos de recoger veremos que nos convierte en cuestionable la distinción misma. Puede que la «actitud de la vida cotidiana» y la «actitud del teorizar científico» sean diferentes, peto no por las descripciones que nos proporcionan Schutz-Garfinkel. En primet lugar, nos preguntamos por el criterio en que se basa esta descripción de las diferencias entre ambas. No lo hemos encontrado, y pensamos que no lo encontraría ni el mismo Aristóteles si para ello sólo volviera. La distinción consta de cinco puntos y podía muy bien, por el camino que ha escogido, llegar a los 15 o a los 20 puntos, o a los que se quisiera. Puestos a describir sin criterio lógico o empírico alguno, a to mejor salía hasta una novela.

Vamos a concentrarnos en los puntos en que la evidencia es contraria a los puntos de distinción de Schutz-Garfinkel. Respecto del primer punto, no es cierto que el teorizar cientifico no reconozca «las estructuras normativas como condiciones que constriñen». Más bien, la teoría sociológica actual, muy particularmente a partir de Durkheim, se ha basado en el postulado de la «contrainte», es decir, de que la realidad social está constreñida por una normativa social, lámese «representaciones colectivas», «valores» e incluso «normas» (por supuesto, no necesariamente, normas jurídicas o morales). Recordemos, dentro de los textos de este trabajo, las «reglas empíricas» de Weber. ftem más, estas normas sociales están vigentes en la vida cotidiana también. De modo que el teorizar científico no hace sino proponer como hipótesis que tales y tales normas están vigentes en una sociedad dada, y la investigación trata de comprobar si ello es cierto o no. La distinción descrita en este punto primero no logra efectivamente marcar una diferencia real entre la vida cotidiana y el teorizar científico. Insistimos en que estamos seguros de que son dos cosas diferentes, pero no por esta razón.

El segundo punto marca una diferencia evidente. Una cosa es saber a qué atenerse en la vida cotidiana, sin lo cual sería imposible «convivir», y otta muy distinta que un científico proponga, por ejemplo, una hipótesis sobre algún aspecto de la realidad social, de modo que hasta que no esté probada la hipótesis el científico renuncie a su conocimiento de «sentido común» y espere a que se confirme el conocimiento científico. Pero esta distinción es lo que se llama en metodología un «truismo». Lo extraño es que parece que Schutz-Garfinkel se sorprendan de que semejantes verdades de perogrullo existan. $\mathrm{Y}$ además - como veremos después- que se le otorgue a estas distinciones, así formuladas, un status lógico que sencillamente no tienen.

EI tercer punto es irrelevante, y además creo que el reloj corre igual 
para la vida cotidiana que para el teorizar científico. No hay dos relojes distintos para cada uno de ellos.

En el cuarto punto se dice que «el teórico se encuentra fuera de un rol». Por lo pronto se encuentra en el rol de teórico, que en las sociedades civilizadas es un rol perfectamente admitido. ¿Cómo seguir leyendo a Schutz-Garfinkel si como teóricos se encuentran fuera de un rol? $O$ es que acaso por maravilla a ambos se les ha concedido la gracia de contem. plar el teorizar científico como individuos de la vida cotidiana... Esta es una decisión que tendrían que haber tomado antes de teorizar - suponiendo que lo que hacen es teorizar-: o hablar como teóricos o hablar como habitantes de la vida cotidiana. Y ésta es una pregunta clave: si la vida cotidiana es tan diferente del teorizar científico, cómo ellos, teóricos supongo, han alcanzado esa penetración en la vida cotidiana. La respuesta tiene que ser; fueza de cualquier rol, ya sea el de la vida cotidiana, ya sea el del teorizar científico. Lo que pasa es que como la diferencia existe, aunque no va tan lejos como suponen Schutz-Garfinkel, desde una perspectiva de teorizar científico se les ha dado el comprender la vida cotidiana. La descripción que ofrecen de la vida cotidiana la hacen desde su perspectiva de teóricos, que no comporta evidentemente las limitaciones que ellos imaginan.

Respecto del quinto y último punto, que un teórico tenga como punto de referencia lo que Ilamarímos el «mundo acadérnico», me parece tan obvio como que en la vida cotidiana se tiene en ctrenta al vecino. Lo raro sería que el científico buscase la aprobación del hombre común, y la mujer de su casa quisiera ser respaldada por un teórico científico.

Este conjunto de obviedades y falsedades o verdades a medias, en torno a un problema como es el de que la "actitud de la vida cotidiana» y la «actitud del teorizar científico» son diferentes, y la descripción medianeja que se hace de las dos cosas, pudiera admitirse como entretenimiento literario, si los autores no pretendieran nada menos que lo siguiente: «Estos dos conjuntos de presuposiciones no se solapan, ni son distinguibles en grado. Más bien, traspasar del uso de un conjunto al otro - -desde una "actitud" a la otra- produce una alteración radical en las estructuraciones de los acontecimientos de las personas y sus relaciones. En el sentido matemático literal las dos actitudes producen conjuntos de acontecimientos lógicamente incompatibles» (pág. 65). Si esto es así, ¿desde la perspectiva de qué conjunto se ha hecho esa descripción? Parece que desde el del teorizar científico. Ahora bien, si los dos conjuntos son lógicamente incompatibles, ¿cómo ha sido posible que Schutz-Garfinkel nos describan el conjunto de la vida cotidiana desde el conjunto del teorizar científico?

Volvemos a decir que estamos de acuerdo en que la «actitud de la 
vida cotidiana» y la «actitud del teorizar cientifico» son cosas diferentes. No discutimos si los cinco puntos se solapan o no. Pero lo que sí discutimos es que no se trate de una diferencia de grado. El hombre que vive en la vida cotidiana es, en primer lugar, el mismo que puede ser teórico. En segundo lugar, la experiencia de la vida cotidiana sirve al teórico como materia prima sobre la que elaborar, por ejemplo, sus hipótesis. En tercer lugar, los mismos procedimientos de la ciencia, entre los que se cuenta el de teorizar, no son incompatibles con la vida cotidiana: más bien, lo que la ciencia hace es «corregir», en gracia de la objetividad, el uso de los sentidos para llegar a poseer un conocimiento científico. Es lo que hemos dicho antes sobre la «observación», como utilización de los sentidos ayudados de medios términos, para así ampliar las posibilidades de nuestros sentidos y corregir posibles prejuicios subjetivos. Pero los sentidos utilizados en la vida cotidiana y en la observación cientítica son los mismos. De modo que se puede concluir que la diferencia es precisamente una distinción de grado.

Lamentamos, eso sí, la ocasión perdida de fundamentar una descripción de la vida cotidiana frente a lo que podemos llamar la vida en la sociedad global. Esta distinción aparece en ia obra de Ortega y Gasset, El bombre y la gente (Madrid: Revista de Occidente, 1957), como separación de lo que él llama «relaciones interpersonales» y «hechos sociales». Ortega entiende que las primeras no son sociales, y que el objeto de la sociología son los «hechos sociales», caracterizados por su carácter de "contrainte». En la actualidad, este problema parece como si lo estuvieran rondando muchos esfuerzos teóricos y empíricos, que no acaban de plantear adecuadamente la distinción. Como pregón de urgencia debo decir que yo también estoy trabajando sobre ello y que, en este sentido, me interesa la línea Schutz-Garfinkel, así como la de Irving Goffman (sobre este último léase la sagaz y temprana en reconocer el futuro de esta línea obta de Amando de Miguel, Introducción a la sociología de la vida cotidiana, Madrid: Edicusa, 1974). Personalmente, pienso que la distinción de Ortega debe ser el punto de partida, pero que hay que considerar a ambos términos de la distinción - «relaciones interpersonales» y «hechos sociales»- como sociales. Aquí no puedo detenerme en fundamentar esta proposición.

Como recordará el lector, el texto de Garfinkel consistía en un paper preliminar teótico para un proyecto de investigación empírica. Bien. Después de haber desarrollado la distinción entre «actitud de la vida cotidiana» y «actitud del teorizar científico», que era la «crux», ta qué conclusiones operativas se Hega?

A la siguiente: «Ninguna necesidad dicta que se decida sobre una definición de acción racional al objeto de concebir un campo de aconte- 
cimientos observables de la conducta. Este resultado tiene la consecuencia importante y paradójica de permitirnos estudiar las propiedades de la acción racional de un modo más cercano que nunca antes... En lugar de que las propiedades de racionalidad sean tratadas como un principio metodológico para interpretar la actividad, ellas son tratadas sólo como material empiricamente problemático. Ellas tienen el status sólo de datos y se tiene que dar cuenta de ellos de la misma manera que se tiene que dar cuenta de las propiedades más familiares de la conducta» (pág. 70; subrayado de H. G.).

Es decir, que al intentar Garfinkel reintroducir la categoría de «tacionalidad» en la investigación social, concluye después de no pocas inútiles disquisiciones - ahora no podemos dejar de verlas así-, que la racionalidad hay que buscarla empíricamente en los datos de la realidad, incluidos los de la vida cotidiana, sin previa definición de lo que hay que buscar. La racional babla por sí misma. Todo consiste en ir a los datos y esperar a que bablen. En la ciencia, a Ia realidad - como dice Zubiri- bay que saber preguntarle. La realidad, para el científico, es muda, y sólo habla cuando se la interroga adecuadamente, es decir, por lo menos -en el caso que estamos tratando-, una definición de racionalidad. De nada sirven los 14 sentidos de la racionalidad elaborados por Schutz, si nos se les convierte en hipótesis, tipo-ideales, definiciones operatorias, instrumentos de observación, diseños de investigación, técnicas de recogida de datos, procedimientos de análisis, etc. Sin todo eso, lo no importante, no paradófico, que ocurre es que no se llega a ninguna parte.

Algo de esto debe tener Garfinkel en mente cuando dice que: «La necesidad inmediata consiste en tener un instrumento que pueda ser usado para estimar los agrupamientos de las racionalidades y su extensión para las acciones de las personas que se encuentran en lugares estratégicamente diferentes de la estructura social» (pág. 71). El proyecto a que se refiere este paper fue redactado en 1960 . Todavía estamos esperando el instrumento en cuestión.

José JimÉnez Bianco

Departamento de Sociologia

Universidad Autónoma de Madrid

Cantoblanco, Madrid 